\title{
El estudiantado colombiano y la apuesta por el cogobierno y la autonomía universitaria (1971-1972): análisis retrospectivo con base en el Manifiesto de Córdoba*
}

\author{
ÁlVARO ACEVEDo TARAZONA** \\ ANDRÉS DAVID CORREA LUGOS***
}

Artículo de revisión sobre la situación del estudiantado colombiano entre los años de 1971 y 1972, la solicitud de autonomía universitaria y el establecimiento de un cogobierno, bajo la retrospectiva del Manifiesto de Córdoba (Argentina) promulgado en 1918.

Recibido: 7 de octubre de 2018 - Evaluado: 6 de diciembre de 2019 - Aceptado: 2 de junio de 2020

Citar como: Acevedo, A. y Correa, A. (2021). El estudiantado colombiano y la apuesta por el cogobierno y la autonomía universitaria (1971-1972): análisis retrospectivo con base en el Manifiesto de Córdoba. Hallazgos, 18(35), 343-370. https://doi.org/10.15332/2422409X.4438

* Resultado parcial del proyecto de investigación "Crónica del 68: live fast die young” (código UISVIE: 2409), financiado por la Universidad Industrial de Santander, Colombia.

** Ph. D. en Ciencias de la Educación. Profesor titular de la Universidad Industrial de Santander, Colombia.

Correo electrónico: acetara@uis.edu.co ORCID: https://orcid.org/0000-0002-3563-9213

** Candidato a magíster en Historia. Investigador Junior Minciencias. Miembro del Grupo de Investigación Políticas, Sociabilidades y Representaciones Histórico-Educativas.

Correo electrónico: andrescorrealugos@outlook.com

ORCID: https://orcid.org/0000-0002-6477-8001 


\section{Resumen}

Este artículo rastrea la evolución del movimiento estudiantil colombiano, el cual se deriva del sentimiento del estudiantado latinoamericano por obtener la autonomía universitaria. El estudiantado colombiano dirige su acción colectiva hacia la búsqueda de espacios de participación en la toma de decisiones de la universidad pública, hasta lograr la implementación del cogobierno en la Universidad Nacional de Colombia, en la Universidad de Antioquia y en la Universidad Industrial de Santander. Es el Manifiesto de Córdoba el documento de ruta para comprender el anhelo de los jóvenes colombianos de implementar nuevas formas de gobierno que les permitan vivenciar una universidad autónoma, libre y democrática. La implementación de los gobiernos universitarios en Colombia, como consecuencia de la Reforma de Córdoba, es el tema central de este artículo. Para ello, en dos apartados, el artículo permite la reflexión acerca de la Reforma de Córdoba y su influjo en el movimiento estudiantil colombiano, el cual se radicalizó en los años setenta. Esta reflexión se obtiene tras la consulta de documentos y periódicos de la época, así como de la reciente historiografía escrita, elementos que permiten rastrear las inflexiones y la trascendencia del movimiento estudiantil colombiano a escala latinoamericana.

Palabras clave: Autonomía universitaria; Cogobierno universitario; Consejo superior; Movimiento estudiantil; Reforma de Córdoba; Universidad colombiana. 


\title{
The Colombian student body and the commitment to co-governance and university autonomy (1971-1972): a retrospective analysis based on the Córdoba Manifesto
}

\begin{abstract}
This article traces the evolution of the Colombian student movement, which derives from Latin American students' feeling for university autonomy. The Colombian student body directs its collective action towards the search for spaces of participation in the decision-making process of the public university, until achieving the implementation of co-governance in the National University of Colombia, the University of Antioquia and the Industrial University of Santander. The Córdoba Manifesto is the road map for understanding the desire of young Colombians to implement new forms of government that will allow them to experience an autonomous, free and democratic university. The implementation of university governance in Colombia, as a consequence of the Cordoba Reform, is the central topic of this article. To this end, in two sections the article allows for reflection on the Cordoba Reform and its influence on the Colombian student movement, which was radicalized in the 1970s. This reflection is obtained after consulting documents and newspapers of the time, as well as recent written historiography, elements that allow us to trace the inflections and the transcendence of the Colombian student movement on a Latin American scale.
\end{abstract}

Keywords: University autonomy; University co-governance; Higher Council; Student movement; Córdoba Reform; Colombian university.

\section{Os estudantes colombianos e a aposta pela cogovernança e pela autonomia universitária (1971-1972): análise retrospectiva com base no Manifesto de Córdoba}

\section{Resumo}

Este artigo investiga a evolução do movimento estudantil colombiano, o que é derivado do sentimento dos estudantes latino-americanos por obter a autonomia universitária. Os estudantes colombianos direcionam sua ação coletiva à busca de espaços de participação na tomada de decisões da universidade pública, até conseguirem a implementação da cogovernança na Universidad Nacional de Colombia, na Universidad de Antioquia e na Universidad Industrial de Santander. O Manifesto de Córdoba é o documento guia para compreender o desejo dos jovens colombianos de implementar novas formas de governo que lhes permitam vivenciar uma universidade autônoma, livre e democrática. A implementação dos governos universitários na Colômbia, como consequência da Reforma de Córdoba, é o tema central deste artigo. Para isso, em duas seções, o artigo permite a reflexão sobre a reforma de Córdoba e sua influência no movimento estudantil colombiano, o que foi radicalizado nos anos 1970. Esta reflexão é obtida após a consulta de documentos e jornais da época, bem como da recente historiografia escrita, elementos que permitem investigar as inflexões e a transcendência do movimento estudantil colombiano no contexto latino-americano.

Palavras-chave: Autonomia universitária; Cogovernança universitária; Conselho superior; Movimento estudantil; Reforma de Córdoba; Universidade colombiana. 


\section{Introducción}

El aspecto que ha caracterizado al estilo de administración universitaria en Latinoamérica es la conformación de tipo gubernamental de los consejos superiores, pues a diferencia de lo que sucede en otras latitudes, en los consejos universitarios latinoamericanos intervienen autoridades gubernamentales externas a la universidad como los ministros, los gobernadores o los alcaldes (Borrero, 2008). Debido a esta característica, en las universidades latinoamericanas el ejercicio efectivo de la administración ha sido objeto de fuertes críticas por parte del estudiantado y el gremio profesoral. Impulsados por diferentes ideologías e intereses políticos y sociales en aras de mejorar su posición en el medio académico, estos grupos han querido modificar en varias ocasiones la composición del principal órgano de gobierno universitario, así como las funciones y prerrogativas de sus miembros. Históricamente, esta pretensión articuló las demandas gremiales con determinadas posiciones políticas con el objeto de obtener el control de los mecanismos del poder universitario, razón por la cual los distintos actores sociales y políticos se han enfrentado en una larga pugna por mantener o acceder al control de las instituciones de educación superior.

No han sido pocas, por lo tanto, las convulsiones administrativas, académicas y sociales que estas instituciones han experimentado a lo largo de su historia. Sin duda, para el caso latinoamericano, los episodios más significativos se presentaron hacia 1918, con la Reforma de Córdoba, y en los años setenta, cuando las ideologías de izquierda "propugnaron no solo por la modificación radical de los consejos superiores, sino incluso por la eliminación de toda autoridad" (Acevedo y Villabona, 2015, p. 148). Si bien este fenómeno histórico se presentó en la mayoría de países latinoamericanos, no deja de haber particularidades elocuentes.

Mientras en Chile, Uruguay y Colombia los movimientos pudieron adelantar, cada uno a su manera, cambios estructurales en la administración universitaria al instaurar sus propios procesos de cogobierno, a los movimientos estudiantiles de México, Argentina, Ecuador y Brasil les fue imposible poner en práctica los cambios administrativos deseados, debido al accionar de los gobiernos dictatoriales o autoritarios de la época.

El problema del gobierno universitario y los intentos de transformación por parte de estudiantes y docentes en el siglo xx estuvieron íntimamente ligados con la constitución de la autonomía universitaria y, por tanto, con el papel de la universidad en los procesos de modernización de las sociedades latinoamericanas. Como bien lo sugiere Múnera, estos dos aspectos de la vida universitaria latinoamericana 
fueron de la mano con la ampliación o restricción de la democracia política y social en cada país. En el transcurso del siglo anterior, la vida de la universidad pasó de una posición que reivindicaba la independencia respecto a los partidos políticos y el Estado a una defensa de la intervención por parte de las instituciones políticas como forma de resistir las determinaciones provenientes de la economía de mercado (Múnera, 2011).

En tal sentido, el objetivo de este artículo es analizar las circunstancias históricas en las que el movimiento estudiantil colombiano de principios de los años setenta luchó por modificar la más alta instancia del poder universitario, el Consejo Superior, con el fin de instaurar un sistema de cogobierno. Escudriñar el momento histórico en que la idea de un gobierno de estudiantes y profesores se hizo posible permitirá conocer cuál fue el impacto mediato de los principios de la Reforma Universitaria de Córdoba (1918) en Colombia, e identificar las posiciones, argumentos, convergencias

y desencuentros de unos jóvenes radicalizados que, producto de la época, consideraron que era posible iniciar la toma del poder empezando por la transformación de las instancias decisorias en sus casas de estudio.

\section{Autonomía universitaria como síntoma o Zeitgeist}

La autonomía es un tema recurrente por parte de los teóricos sociales al analizar las voluntades colectivas en medio de coyunturas de represión, o de oportunidades y posibilidades políticas. Sin embargo, al ser coyunturas en ocasiones disímiles, es posible que el concepto tienda a diluirse o vulgarizarse. Esto lleva a que en muchas ocasiones se aborde el tema sin definir siquiera lo que concibe la autonomía. Además, la autonomía es un concepto sintomático, es decir, no es constante y sus usos varían entre las finalidades políticas, la ideología o inclusive las vías que toman los actores sociales, violentas o no violentas, para defenderla. Incluso dentro de un mismo sector poblacional - por ejemplo, los universitarios - o dentro de un territorio como la universidad, la autonomía puede tener distintas concepciones. Esta particularidad polisémica enriquece los estudios sociales, pues no solo abarca el análisis de una coyuntura sino el uso de un concepto de larga duración en intervalos cronológicos cortos pero cambiantes.

La propuesta de este artículo es revisar el concepto de autonomía como una muestra sintomática de un malestar social. Para abordarlo como síntoma, es conveniente remontarse a la conceptualización hegeliana del Zeitgeist, un término de 
origen alemán que significa el "espíritu generacional” o de la época. Se usa para referirse a todas aquellas coyunturas y acontecimientos que marcan una inflexión en una atmósfera social determinada y delimitan la forma como las personas conciben las necesidades de su tiempo y toman decisiones, y es retomada de Goethe al definirla como: "Lo que llamas el espíritu de los tiempos es, en el fondo, el espíritu de las gentes en quienes los tiempos se reflejan” (Goethe, 1806, p. 133). La importancia de la conceptualización de Zeitgeist para el análisis de la autonomía universitaria reside en que este constituye una reclamación por parte de la masa, no de sectores que ostentan el poder; entonces, la mayoría de veces esta esencia generacional aparece cargada de desencanto, lo cual da pie a las protestas sociales, y la autonomía surge aquí como una estrategia para legitimar la inconformidad dentro de entornos como la universidad.

Uno de los mejores balances sobre la autonomía universitaria es el propuesto por Leonardo Vaccarezza (2006). En primera instancia, el autor enfatiza que la autonomía refuerza la identidad de los integrantes de las instituciones universitarias. Ahora bien, al estar enmarcada en un contexto de tensión entre la universidad y el Estado, la autonomía es un vector que mide la intensidad de las fuerzas entre los objetivos de los grupos de poder y las instituciones educativas. A raíz de esta tensión en las funciones y los poderes, la autonomía se erige como una reivindicación frente a las pretensiones de represión y hace que las universidades se conviertan en símbolo contra los gobiernos y las reformas de turno.

Sin embargo, la noción de autonomía se torna problemática, en especial en las universidades de carácter público, cuando los objetivos propios de la institución son distintos a los del Gobierno y parte de los recursos con los que se sostienen las universidades dependen del Estado. Este problema de la autonomía se debe a su naturaleza jurídica, pues se puede concebir como la capacidad que tiene un ente para darse su propia ley y regirse por ella (Finocchiaro, 2004). Para el caso latinoamericano, la academia se apropia de la autonomía como herramienta que pretende redefinir también a la misma universidad al pasar de ser una institución que consolida y sostiene el orden de las élites para comprometerse con causas sociales, en especial la desigualdad.

Más aún, como la mayoría de los conceptos, la autonomía universitaria depende de los actores que hacen uso de ella: por ejemplo, los docentes defienden la libertad de cátedra; y los administrativos, que la toma de decisiones no dependa del Estado; para el estudiantado, esta se representa en la toma democrática de decisiones dentro de la universidad. Por ello, la autonomía universitaria, al igual que 
la individual, es una cuestión del ambiente interior que se proyecta en el exterior y enlaza la vida interna de la universidad en relación con el Estado y la sociedad (Vaccarezza, 2006). En otras palabras, la autonomía es una cuestión de voluntad, que parte desde el sujeto y de la introspección de aquello que considera justo y necesario. Si bien la autonomía universitaria cambia por las necesidades y consideraciones desde la voluntad de los individuos y colectivos, para Rébora (1989), existe un componente latente y constante en la proyección social, el sentido americanista y sus fundamentos antioligárquicos y antiimperialistas; debido a ello Vaccarezza concluye:

La autonomía universitaria en América Latina se constituye, para el imaginario político de sectores importantes, en un concepto que se articula en luchas que trascienden el espacio académico y se proyectan en el plano de la transformación social. Este significado ampliado de autonomía se expresa a lo largo de la experiencia latinoamericana en la extensión social como una función fundamental de la universidad, planteada más allá del mero servicio, actualización o articulación con la industria cultural como eje de transformación a través del servicio del saber a la lucha social. (Vaccarezza, 2006, p. 38)

En síntesis, el concepto de autonomía universitaria hace énfasis en una cuestión de voluntad que inicia en cada uno de los individuos y colectivos y reafirma las identidades, pero está sujeta a los cambios y a lo intempestivo de las coyunturas sociales. Ahora bien, la tensión pendular de la relación entre Estado y universidad hace que subsistan fricciones, las cuales muchas veces desencadenan encuentros violentos y polarización de discursos dentro de los campus y fuera de estos.

Finalmente, es importante resaltar su papel protagónico en la construcción de culturas políticas dentro de la comunidad universitaria, las cuales pueden ir en sintonía o en contra de los planes gubernamentales, sin embargo, la autonomía universitaria es uno de los pocos ejercicios autonómicos que ofrecen un espectro de decisiones y propuestas a la par de las posiciones que hay dentro de la universidad, lo cual hace que muchas veces se difumine el concepto. 


\section{De la Reforma de Córdoba a la radicalización del movimiento estudiantil ${ }^{1}$ en Colombia, 1910-1957}

La idea de la participación estudiantil y profesoral en la administración de las universidades retomó los principios de la democracia representativa que fueron postulados por primera vez durante la Reforma de Córdoba en 1918 (Sader, Gentili y Aboites, 2008). No obstante su carácter liberal y trascendencia a lo largo del siglo xx, fueron los cambios demográficos, especialmente la urbanización de las ciudades latinoamericanas, el crecimiento económico y la inserción de las juventudes como un nuevo actor social, los que incidieron en la actualización de las demandas por cambiar la estructura de poder de las instituciones universitarias entre los años sesenta y setenta. Como se verá más adelante, a ello habría que sumar la fuerte ideologización de los estudiantes, que los llevó a plantearse la discusión en torno al papel que le correspondía a la universidad en la transformación de la sociedad.

En Colombia, las primeras acciones del movimiento estudiantil se presentaron entre 1910 y 1957, año de finalización del gobierno del general Rojas Pinilla (Archila, 1999). Sin duda, estas manifestaciones estuvieron influenciadas por el movimiento reformista de Córdoba, el cual sentó las bases de la lucha estudiantil al propender no solo a la modernización de la educación superior, sino también a la búsqueda de la autonomía universitaria, un elemento que prevalecerá a lo largo de toda la historia de la protesta estudiantil (Ocampo, 2008). Con un panorama educativo deplorable luego de la guerra de los Mil Días, los estudiantes universitarios de las tres primeras décadas del siglo xx ejercieron la reivindicación de la educación como medio para acometer reformas políticas y sociales que contribuyeran a redimir la nación. El mensaje de ligar la universidad a la realidad social caló hondo entre los jóvenes universitarios del continente y, sin duda, se convirtió en el acicate para la movilización por la reorganización y la redefinición de la universidad (Acevedo, 2010).

El influjo del Manifiesto de Córdoba en Colombia se expresó de diversas maneras y con ritmos particulares. Al igual que en otras universidades latinoamericanas, la educación superior en Colombia proscribía la literatura más avanzada del racionalismo moderno, los métodos de estudio eran caducos y se mantenían los ritos de

1 A pesar de sus especificidades, la presencia continua del estudiantado en el escenario de la protesta social, sus experiencias organizativas, la pervivencia de sus demandas y la constitución de una identidad y un proyecto societal —especialmente en las décadas de estudio- son los elementos que permiten hablar de movimiento estudiantil. 
la profesionalización (Ciria y Sanguinetti, 1987). Sin embargo, en nuestro contexto las clases medias como sector social, con sus propias demandas de educación y capital cultural, no surgirían sino hasta mediados de siglo. Por esta razón, fueron los estudiantes provenientes de la misma élite social, política y económica los que acometieron los primeros intentos organizativos, impulsaron las conexiones con los discursos y problemáticas continentales y promovieron una tímida modernización de la universidad colombiana a partir de la lectura y apropiación de documentos como el Manifiesto Liminar de Córdoba (Cantón, Moreno y Ciria, 2005; Buchbinder, 2005; Pastrana, 2008).

En resumen, las demandas contempladas en el Manifiesto, redactado por el abogado Deodoro Roca, planteaban principalmente la libertad de cátedra, la libertad para el estudio de cualquier tipo de ideas, la obligación de involucrar a la universidad con la búsqueda de soluciones a los problemas sociales, la vinculación de la universidad con el resto del sistema educativo nacional, la asistencia libre a clases, la gratuidad, la obligación de la universidad para buscar la unidad latinoamericana, la lucha contra cualquier forma autoritaria de gobierno y, sobre todo, la autonomía y el cogobierno universitarios (Sader, Gentili y Aboites, 2008). Además, presentaban una fuerte resistencia a las intervenciones extranjeras, en especial, al dominio de Estados Unidos en la implementación y guía de la universidad latinoamericana.

Los alcances en Colombia de este movimiento reformista se sintieron en las discusiones sobre la autonomía universitaria y la posibilidad — remota en la primera mitad del siglo - de obtener injerencia en el gobierno universitario (Borrero, 2008) ${ }^{2}$. Producto de este contexto internacional y de acuerdo con las condiciones nacionales de agotamiento de los diferentes gobiernos, entre 1929 y 1957 se presentaron en el país diversas protestas estudiantiles. Los acontecimientos más importantes fueron, indudablemente, la celebración del Primer Congreso Internacional de Estudiantes de la Gran Colombia en 1910, las protestas de 1929, la movilización de mayo de 1938, las pedreas de 1946 y las movilizaciones que acompañarían la caída de la dictadura de Rojas Pinilla en 1957. Estos movimientos universitarios estuvieron motivados por el afán de intervenir en la vida pública del país en el marco de una relación ambivalente con los partidos políticos tradicionales; la bandera de la autonomía universitaria

2 Borrero Cabal es muy crítico respecto al influjo de la Reforma de Córdoba en Latinoamérica. En todo caso acepta que el cogobierno fue el aspecto más reivindicado por los movimientos universitarios latinoamericanos subsecuentes, pero indicando que su aplicación resultó negativa para el desarrollo de la universidad. 
no fue motivo de ruptura con el establecimiento político sino solamente cuando las circunstancias globales cambiaron.

El Primer Congreso Internacional de Estudiantes de la Gran Colombia, Ecuador y Venezuela se realizó en julio de 1910 y estuvo protagonizado por los jóvenes que más adelante serían conocidos como los miembros de la Generación del Centenario, entre los que se destacaron Agustín Nieto Caballero, Tomás Rueda Vargas, Pablo Vila, Eduardo Santos, Luis Cano y Miguel Fornaguera, entre otros (Henderson, 2006). En un contexto de republicanismo representado por la presidencia de Carlos E. Restrepo, y con una economía cafetera en despegue, los estudiantes centenaristas preconizaron la conciliación como divisa para conseguir el progreso del país (Arias, 2007). Para ello, crearon la Revista Universitaria, como medio de difusión de su ideario reformista, y fueron los organizadores de dicho evento internacional estudiantil como espacio para discutir temas como la participación y la representación estudiantil dentro del sistema universitario o la creación de una asociación estudiantil en Colombia (Salgado, 2015). El programa de esta organización estudiantil corrió por cuenta de Luis López de Mesa y recogió algunas de las inquietudes del reformismo de Córdoba (Castro, 2007).

En octubre de 1924, Germán Arciniegas y otros jóvenes universitarios exigieron que se reconociera la autonomía de la Universidad Nacional de Colombia y se diera participación a los estudiantes en el gobierno universitario (Acevedo y Correa, 2016)3. A través de la creación de nuevos órganos informativos — como Voz de la Juventud y Universidad, ambos dirigidos por Arciniegas-, en los años veinte se dieron nuevos intentos de organización estudiantil que reclamaron la autonomía de la universidad colombiana y la formación de espacios culturales que dotaran a los estudiantes de una identidad, sin menoscabo de la crítica al sistema educativo (Salgado, $2015)^{4}$. Como parte de este proceso de organización, y en un marco de efervescencia social contra el gobierno de Abadía Méndez, la protesta estudiantil de 1929 fue la

3 En Scott y Lyman (1974) se lee: "La universidad que nosotros buscamos — decían Arciniegas y sus amigos- $-[. .$.$] ha de ser una universidad independiente. Dueña de sus propios recursos, libre$ de toda tutela, que pueda orientarse en cualquier instante de acuerdo con las sugestiones de la ciencia y de la vida. Reflejo del alma nacional, contradictoria e inquieta, pero jamás del poder exclusivo de un poder político. Que se desenvuelva su organización con autonomía verdadera, que se dicten sus leyes por el acuerdo entre el profesorado y los estudiantes, que jamás pueda turbar su marcha el temor de una imposición extraña” (p. 270).

4 Una iniciativa interesante que no se volvió a repetir en los estudiantes colombianos fue la creación de un Centro Universitario de Propaganda Cultural (Pulido, 2010). 
El estudiantado colombiano y la apuesta por el cogobierno y la autonomía universitaria...

manifestación pública más importante de la hasta ese momento breve historia del movimiento estudiantil colombiano, no solo porque produjo el primer mártir del estudiantado colombiano sino por la incidencia que tuvo en la caída del dominio conservador (Acevedo, 2017).

A finales de los años treinta, una nueva generación de estudiantes se dispuso a encarar el reto de fortalecer su presencia en la esfera pública nacional a través de demandas gremiales que, poco a poco, abrieron paso a un nuevo tipo de relación con el establecimiento político. El paro estudiantil de mayo de 1938, iniciado en Medellín y rápidamente propagado al resto del país, fue motivado por un examen de revisión y un curso preparatorio, circunstancia que sirvió para expresar la molestia estudiantil al ver amenazada su movilidad social. La protesta no solo recibió el respaldo inmediato de los estudiantes universitarios, sino que fue aprovechada para ampliar la gama de demandas, exigiendo no solo nuevos profesores, rebajas en las matrículas, la creación del servicio de salud, la libertad de cátedra y de asistencia a clases, sino la puesta en marcha de "una nueva organización de la universidad" (Moreno Martínez, 2009, p. 47).

A partir de 1946 se sentaron las bases para el surgimiento de una protesta estudiantil cualitativamente distinta con relación a las de años atrás. En primer lugar, se iniciaron los conflictos directos con las fuerzas policiales, surgidos en el marco de un arrasamiento de los sistemas morales que permitió el empleo indiscriminado de la violencia pública y privada para dirimir conflictos sociales (Quiroz, 2002; Palacios, 2012). La violencia bipartidista y estatal generó una reacción igualmente violenta por parte de algunos sectores sociales, entre los que se destacaron los estudiantes, quienes protagonizaron una quinta parte de las protestas que se registraron entre 1948 y 1972 (Archila, 1999). Los estudiantes se asumieron como representantes de una intelectualidad perteneciente a las nuevas clases medias en ascenso, condición que les hizo una de las colectividades más sensibles a los recortes democráticos que se vivieron en los años de la dictadura de Rojas Pinilla (Archila, 1995).

Los sucesos del 8 y 9 de junio de 1954, en los cuales murió casi una decena de estudiantes cuando marchaban para conmemorar un aniversario del asesinato de Gonzalo Bravo Pérez, ocurrido en 1929, pueden ser considerados como el punto de inflexión de una etapa marcada por el conflicto y la desconfianza mutua frente al grupo de poder. En aquella jornada perdieron la vida, además de Uriel Gutiérrez, ocho estudiantes más: Jaime Moore Ramírez, Hernando Morales Sánchez, Hugo León Velásquez, Carlos J. Grisales, Álvaro Gutiérrez Góngora, Elmo Gómez Lucich, Rafael Sánchez Matallana y Hernando Ospina. Esta masacre condujo al movimiento 
estudiantil a buscar nuevos mecanismos de movilización y a cambiar su percepción de las autoridades políticas. Esto explica la creación de la Federación Nacional de Estudiantes Colombianos (FEC), desde la cual se despojaron de la tutela partidista para ejercer una oposición franca contra el régimen de Rojas Pinilla (Ruiz, 2002). Como lo ha señalado Francisco Leal Buitrago, la participación del movimiento estudiantil en los eventos que contribuyeron al derrocamiento de Gustavo Rojas Pinilla fue importante por cuanto sirvió como punta de lanza del descontento civil general, sentando las bases para la radicalización que caracterizaría la movilización estudiantil en las siguientes dos décadas (Leal, 1984).

\section{Movimiento estudiantil y radicalización política: lucha por el cogobierno universitario en Colombia, 1958-1972}

La ampliación de la participación de ciertos estamentos en los órganos de decisión de la universidad constituyó una de las luchas centrales de los estudiantes universitarios en las décadas del sesenta y setenta. El predominio del enfoque antiautoritario proclamado en el principio de extraterritorialidad de la fuerza pública en los campus; el componente académico que promovía una concepción de universidad estrictamente científica, alejada del entorno y los problemas sociales; y la visión económica centrada en la capacidad de autofinanciación, fueron perspectivas de universidad que se situaron en el vórtice de las luchas estudiantiles entre 1958 y 1972 (Acevedo, 2004; 2016). En estos años, todos los actores universitarios y los sectores económicos y sociales, entre ellos los partidos políticos, los gremios y la Iglesia católica, tenían claro que la universidad era un escenario de tensiones, y más aún, un campo de batalla entre múltiples intereses, proyectos de sociedad e ideologías contrapuestas. Ello explica que la lucha por el cogobierno adquiriera tal relevancia.

Las décadas del sesenta y setenta fueron testigos de diversas protestas estudiantiles y juveniles por parte de jóvenes que pertenecían a la generación de posguerra, quienes encabezaron las luchas del Mayo francés, las protestas por los derechos civiles de los afroamericanos, las campañas en contra de la guerra en Vietnam, las críticas al socialismo real o la lucha por los derechos de las mujeres (Acevedo, 2017). En Colombia, el escenario específico en el que se desplegaron las prácticas, los discursos y valores de esta revolución global fue la universidad. Los repertorios de protesta desarrollados por los estudiantes se fundaron en referentes culturales que circularon por el mundo entero, especialmente los relacionados con las diversas corrientes del 
El estudiantado colombiano y la apuesta por el cogobierno y la autonomía universitaria...

marxismo. En consecuencia, dicha protesta conjugó elementos, tales como: la oposición al régimen político, los conflictos generacionales y la crítica al modelo universitario basado en la apuesta desarrollista que Estados Unidos hiciera hegemónica en América Latina (Tirado, 2014; Moreno Durán, 1989) ${ }^{5}$.

Desde el mismo momento en que el Frente Nacional (1958-1974) alcanzó su sanción, el movimiento estudiantil, que venía en alza por su participación en la caída de Rojas Pinilla, emprendió nuevas luchas. Entre 1958 y 1972 las protestas estudiantiles empezaron a radicalizarse debido, entre otros factores, a su acercamiento con otras fuerzas sociales, especialmente las obreras y campesinas, y a una inmersión en los referentes ideológicos que ofrecía la izquierda mundial. Este giro hacia la izquierda fue sin duda uno de los elementos que caracterizó al movimiento estudiantil colombiano durante estas décadas (Archila y Cote, 2009; Acevedo y Samacá, 2013). El influjo de las Revoluciones china y cubana, aunado a la presencia del comunismo soviético, permearon nuevos intentos organizativos que alcanzaron su punto más alto a nivel gremial en la Federación Universitaria Nacional (FUN). Fue allí donde se dieron cita diferentes corrientes políticas e ideológicas que empezaron a liderar las posiciones antiestatales y revolucionarias en las filas estudiantiles (Jiménez, 2000).

La FUN tuvo su origen en medio de la efervescencia juvenil que ayudó a fortalecer la protesta social a principios del Frente Nacional ${ }^{6}$. La articulación de las demandas gremiales con exigencias de cambio político ya venía desde la Unión Nacional de Estudiantes Colombianos (UNEC) a finales de los años cincuenta (Leal, 1984). Más aún, como lo señalan Latorre y Murillo, entre 1958 y 1968 los estudiantes universitarios manifestaron su inconformismo no solo con los partidos políticos tradicionales, sino con el sistema político mismo. Esta desconfianza, más la fuerza que tomó la izquierda en las universidades, ayudan a comprender la radicalización del estudiantado que los llevó a plantearse la posibilidad de tomar el control a pesar de no encontrar un

5 A través de la literatura se puede observar este ambiente cultural en el que se inscribe la lucha por el cogobierno universitario (Samacá y Calderón, 2015).

6 Entre los hechos concretos que incidieron en la creación de esta organización se pueden mencionar: la masacre estudiantil de los días 8 y 9 de junio de 1954 en Bogotá; la golpiza que las fuerzas armadas propinaron a algunos de los asistentes a la plaza de toros La Santamaría en enero de 1956, como reprimenda por abuchear a María Eugenia Rojas, hija de Rojas Pinilla; el enfrentamiento permanente del régimen con la prensa y el posterior cierre de algunos medios como El Tiempo, El Espectador, El Diario Gráfico y El Siglo, que terminó por agudizar el descontento social. 
consenso entre sus filas en torno a este objetivo, producto de la extrema ideologización que padecían (Latorre y Murillo, 1984; Losada y Vélez, 1984; Santamaría, Vélez y Silva, 1984).

La comprensión de la lucha por el cogobierno universitario debe inscribirse, entonces, en el giro hacia la izquierda del estudiantado, asociado a la importancia que adquirieron las clases medias en ascenso, aunado a la cerrazón del sistema político bajo el Frente Nacional y la progresiva desconfianza de la oposición social y política al sistema democrático. A ello podemos agregar el uso de las fuerzas armadas para contener las protestas estudiantiles contra la presencia del presidente Carlos Lleras Restrepo y el filántropo John Rockefeller, lo cual devino en el fin de la FUN en 1966 (Leal, 1984). Estos hechos constituyeron la antesala de la cresta del movimiento estudiantil, que hizo del cogobierno su principal demanda en los años 1971-1972.

La coyuntura de los años 1971-1972 viene a ser la continuidad nacional del movimiento contracultura protagonista del año 1968. La rebeldía siempre estuvo latente, sin embargo, a partir de este año tomaron fuerza conceptualizaciones ideológicas de la nueva izquierda caracterizada por la búsqueda de nuevas experiencias sensoriales, sociales y políticas, algunas basadas en distintas versiones del trotskismo, como la de los conservadores apegados a la revolución programática o la de aquellos que consideraron que el acto de revolución más grande es la rebeldía total, política, cultural y sexual (González, 2018).

A la par que los estudiantes se movilizaban en todo el país contra el despliegue represivo instaurado por el presidente Misael Pastrana, en medio de un estado de sitio que inició en Cali y luego se extendió por todo el país, se produjo uno de los incidentes más recordados de contracultura en la historia de las juventudes colombianas. Entre el 18 y el 20 de junio de 1971 se realizó a las afueras de Medellín el Festival de Ancón. Alrededor de 200 mil jóvenes se desplazaron hasta el municipio de La Estrella con la consigna de rebeldía, liberación sexual y experimentación. Al igual que Woodstock en Estados Unidos, Wight en Inglaterra o Avándaro en México, el Festival de Ancón representó una experiencia pacifista paralela a la radicalización y el conservadurismo. El festival tuvo una gran acogida, pues fue un evento que congregó a un sector nuevo de la población en las ciudades colombianas como eran los jipis.

En una realidad política y social marcada por la violencia y la desigualdad social y un empeño por la modernización a la fuerza, era apenas lógico que los jóvenes buscaran alternativas en sincronía con discursos globales como el amor, la libertad y el respeto por la naturaleza. El Festival de Ancón fue un experimento social de utopía con una duración de 72 horas. Por estos tres días los asistentes se olvidaron del 
El estudiantado colombiano y la apuesta por el cogobierno y la autonomía universitaria...

estado de sitio y la represión. La entrada al festival costaba 13,20 pesos, solo que, debido a la masiva asistencia, terminó siendo gratuito; llegaron jipis de todo el país y del exterior. Al contrario de lo que se piensa, el festival fue avalado por el alcalde de Medellín de ese momento, Álvaro Villegas. En la entrada del festival había un letrero que decía “todo será amor”, una consigna distinta en tiempos de revolución, resistencia y represión (Castro, 2005).

Pero no todo fue amor. En Medellín, centenares de personas se quejaron del festival y tomó la vocería el sacerdote Fernando Gómez, quien dijo en un programa de radio:

El alcalde autorizó a los millares de hippies a que nos invadieran con una arrolladora avenida de fango putrefacto para que abofetearan con sus manos sucias el rostro de la ciudad, para que invitaran a los niños a ser maleducados, ruines, perversos y para que incitaran a la juventud a embrutecerse en el mundo del amor libre y de los estupefacientes destructores y enervantes. La insólita conducta del alcalde lo priva de toda autoridad moral y cívica para continuar rigiendo los destinos de Medellín, la ciudad culta, honorable y digna, espera su renuncia. No le faltará qué hacer en la república de los hippies, donde será acogido por una salva de aplausos y coronado como el rey de la turba delirante de vagos y degenerados que hablan con voz entrecortada, miran con ojos cansados de marihuana y disputan a los animales inmundos el fango y la yerba maldita. (Barriga, 2015, párr. 10)

En el festival no se presentaron desmanes, las cifras de violencia y muertos fueron cero. Sin embargo, una vez concluido, el Departamento Administrativo de Seguridad (DAS) desplegó un operativo para expulsar a los jipis de la ciudad; el alcalde Álvaro Villegas, por presión de las altas esferas, fue destituido. La prensa también sería crítica al mostrar la vida de los jipis dentro del festival, lo cual quedó reflejado en algunos artículos (figura 1).

Ahora bien, detrás de la organización de Ancón estuvo Gustavo "Carolo" Caro, un reconocido estudiante de economía perteneciente al movimiento de los nadaís$\operatorname{tas}^{7}$. Esta corriente de pensamiento fue famosa por el discurso rebelde y la búsqueda de la ruptura con la sociedad y el tradicionalismo colombiano. El contacto con la psicodelia en la década de los setenta se produjo en Bogotá, donde los jóvenes

7 El nadaísmo fue un movimiento que se opuso al ambiente cultural establecido por la academia, la Iglesia y las ideas tradicionales. Fue constituido en los años sesenta por Gonzalo Arango en Colombia. 
capitalinos compartieron con norteamericanos de los cuerpos de paz y con algunos rebeldes que huían de Vietnam. En su primer momento, esta apertura rebelde fue una opción para los jóvenes de las clases media y alta, pues tenían el dinero suficiente para comprar discos en el exterior, viajar y mantener el costo de las drogas. En otras palabras, fue parte de la emulación del sistema a la revolución del 68, que convirtió al revolucionario antisistémico en un ávido y omnívoro consumidor cultural de experiencias rebeldes.



Figura 1. Los “Hippies”: sed, hambre y pantano en el Festival del Ancón.

Nota: artículo publicado en El Colombiano, Medellín, el 20 de junio de 1971, p. 6.

Fuente: Radio Macondo (25 de diciembre de 2018).

La demanda por la transformación de las estructuras de gobierno en la universidad colombiana formó parte de una coyuntura de movilización estudiantil que tuvo su punto álgido en los hechos de febrero de 1971 en la Universidad del Valle. Como respuesta a la injerencia de instituciones norteamericanas en dicha universidad, los estudiantes marcharon por las calles de Cali el 26 de febrero, hecho que provocó una respuesta represiva por parte de las autoridades que arrojó como resultado la muerte de más de quince estudiantes (Ordóñez, 2007; Herrera y Trujillo, 1993), y el consiguiente estado de sitio como mecanismo de control de la situación. El registro periodístico de la figura 2 da cuenta de los hechos ocurridos en el país tras la muerte de los estudiantes en Cali. 


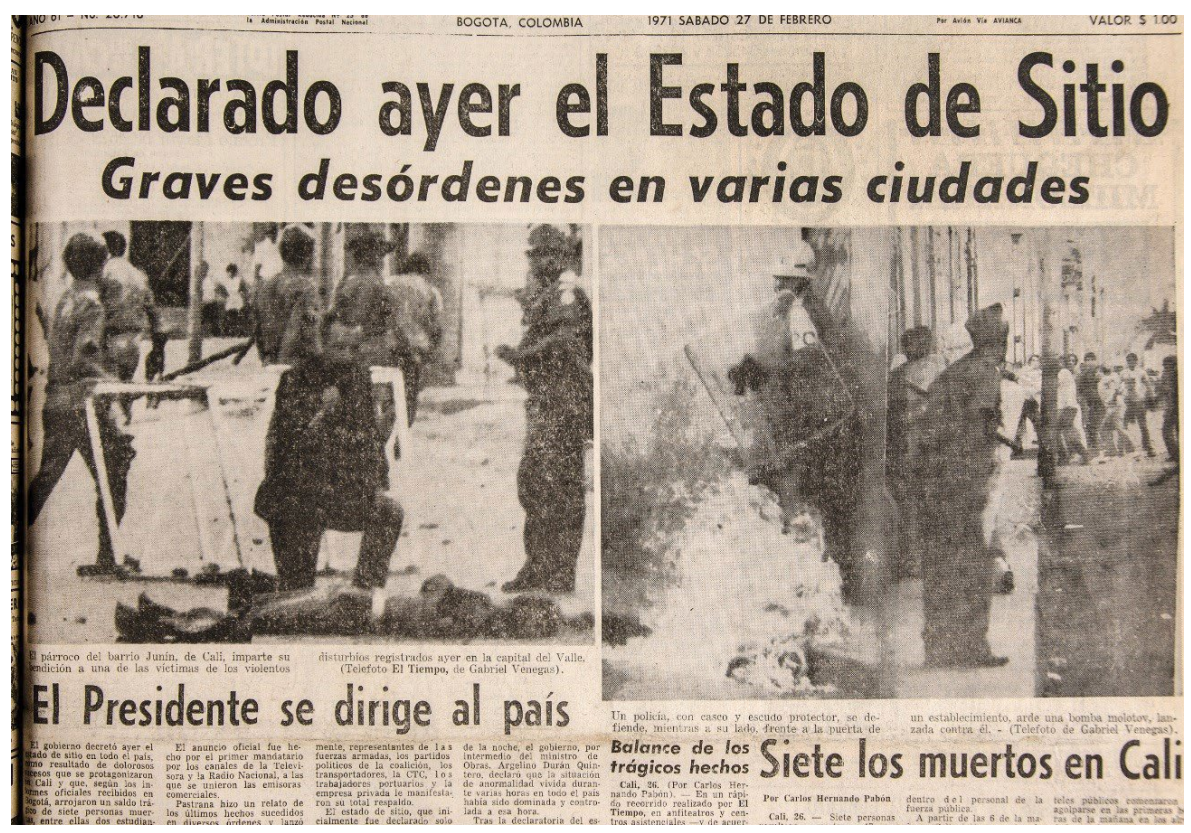

Figura 2. Declarado ayer el estado de sitio.

Nota: artículo publicado en El Tiempo, 27 de febrero de 1971, p. 1. Fuente: Jiménez (2017).

El efecto dominó no se hizo esperar, puesto que las manifestaciones de los estudiantes se precipitaron en diferentes universidades del país, principalmente en ciudades como Bogotá, Bucaramanga y Medellín, paralizando las instituciones de educación superior y las calles. La respuesta de las autoridades políticas fue la prolongación del estado de sitio por temor a una alteración del orden público de grandes proporciones $^{8}$.

Producto de estos eventos y de los diferentes encuentros estudiantiles que se realizaron entre marzo y diciembre de 1971, y tal como lo hicieron en 1918 los estudiantes argentinos, los universitarios publicaron el Programa Mínimo de los estudiantes colombianos, como resultado del Segundo Encuentro Nacional de Estudiantes Universitarios llevado a cabo en Bogotá entre el 13 y el 15 de marzo, el cual fue rápidamente

8 Respecto a los hechos de Medellín, se puede consultar a Garzón (2010); en cuanto a lo sucedido en Bogotá, los artículos de El Tiempo de 1971. Una mirada general a esta coyuntura se encuentra en Cote (2009). 
adoptado por el movimiento universitario en su conjunto. El documento condensaba las ideas que sobre la universidad planteaba la izquierda estudiantil. Junto con las preocupaciones por la financiación estatal, el intervencionismo estadounidense y las posibilidades de la investigación científica, sobresalía el problema de la administración de las universidades y la toma de decisiones, es decir, la cuestión del gobierno. En respuesta a ello se estructuró como primer punto del Programa la abolición de los Consejos Superiores (Hernández, 2007).

En sus propuestas, los estudiantes plantearon la creación de organismos provisionales de gobierno universitario compuestos por el rector (sin voto), quien lo presidiría, un representante del Ministerio de Educación, tres estudiantes y tres profesores, en representación de sus respectivos estamentos (Programa Mínimo del Movimiento Nacional Estudiantil, 1971). El Programa exigía además la conformación de una comisión nacional que estudiara el proyecto de reforma de la Ley Orgánica de las Universidades, en la que dominaría la representación estudiantil y profesoral. Establecida la hoja de ruta de la lucha estudiantil, el movimiento se dio a la tarea de buscar su aplicación en cada una de las universidades cuyos representantes estudiantiles la habían aceptado. A pesar de las respuestas represivas en diferentes ciudades por parte de la fuerza pública, las autoridades universitarias coincidieron en la necesidad de impulsar cambios administrativos con el fin de modernizar las universidades.

En efecto, entre el 16 y el 17 de octubre de 1970 se desarrolló en Cali la primera reunión de rectores de las universidades oficiales del país como parte de la Asociación Colombiana de Universidades (Ascun). En el evento se trataron temas relacionados con la financiación de la educación superior y la autonomía universitaria. Con respecto al segundo punto, los rectores concluyeron que la universidad venía perdiendo autonomía desde la reforma constitucional de 1968, pues allí se había aprobado que los rectores fueran nombrados o removidos sin mayores dificultades por los representantes estatales. Con base en ello, Ascun le propuso al Gobierno nacional la creación de un nuevo mecanismo para la elección de rectores, en el que tales cargos fueran designados por los organismos universitarios y no por entidades externas a la universidad (Asociación Colombiana de Universidades - Consejo Nacional de Rectores, 1970a). La propuesta no consideraba inconveniente que los organismos colegiados de las universidades contaran entre sus miembros con representantes de instituciones externas, pero también recomendaba la presencia de egresados, profesores y estudiantes, sin que ningún sector alcanzara mayoría decisoria (Asociación Colombiana de Universidades - Consejo Nacional de Rectores, 1970b). 
Aunque, en términos retóricos, el Consejo de Rectores manifestara que su deseo no era otro que el de propiciar una reorganización institucional centrada en la armonización de las relaciones entre los distintos estamentos de la comunidad académica, lo que en realidad estaban haciendo era cuestionar la falta de autonomía con la que hasta ese entonces operaban las universidades colombianas. Por esta vía también se entró a cuestionar la forma como se ejercía el poder en las casas de estudio, posibilitando en cierto modo el cambio de su estructura de gobierno. A pesar de esta convergencia, estudiantes y directivas no lograron abrir espacios de diálogo con los rectores, razón por la cual en cada universidad se dio una salida particular a la aguda coyuntura.

En la Universidad de Antioquia, por ejemplo, para dar solución a la crisis, su consejo directivo inició la discusión en abril de 1971. El asesor jurídico de la Universidad inició los diálogos, señalando que era imposible abolir el Consejo Superior de la Universidad, pues la institución no estaba facultada para ello. Propuso en consecuencia que su estructura fuera simplemente modificada a través de una recomendación del Consejo Directivo. Indicó, además, que dicho cambio debía consistir en ampliar los niveles de representación, incluyendo en el Consejo a los estamentos estudiantil y docente, de manera que se les respetara a los delegados del gobernador, del Ministerio de Educación y de la Iglesia su derecho a formar parte de tal instancia del poder universitario (Administración Documental Universidad de Antioquia, 1971a). Para ese momento los gremios habían renunciado a los consejos superiores en varios lugares del país, mientras que la Iglesia defendía su derecho a participar en estos. Por su parte, los estudiantes y los profesores consideraban que debían ser mayoría, dejando en desventaja así a los representantes del Ministerio de Educación y a los rectores (Uribe Ferrer, 1971; Administración Documental Universidad de Antioquia, 1971b).

Discusiones similares se llevaban a cabo en otras regiones del país. En todas, los profesores coincidían con lo que al respecto expresaba el médico veterinario Óscar Bonilla, representante de los gremios profesionales ante el Consejo Superior de la Universidad de Antioquia, cuando afirmaba públicamente:

Creo que la composición del Consejo Superior Universitario en su concepción actual, como estructura de mando en la Universidad, no es adecuada porque no representa auténticamente a la sociedad [...] he llegado al convencimiento de que esta contribución es más efectiva brindando la oportunidad para la reestructuración del Consejo Superior y facilitando así el tránsito hacia las verdaderas reformas que sean garantía para la conducción de los destinos de la Universidad, [...] 
o se representan los intereses de esas asociaciones y en este caso creo se estaría lesionando la autonomía universitaria o bien no se representan esos intereses y entonces no tiene fundamento la posición [...] mi posición no es una evasiva para enfrentar los problemas sino por el contrario es ponerme frente a la realidad propiciando una solución que espero sea compensada con una verdadera reforma y especialmente con una acertada reestructuración del Consejo Superior Universitario. (García Posada, 1971, p. 1)

En la Universidad Nacional de Colombia la situación no era diferente. Aunque la institución venía experimentando cambios administrativos desde la gestión del rector José Félix Restrepo, su organización se caracterizaba aún por poseer una autonomía restringida y un consejo superior en el que primaba el voto de los representantes del Gobierno y de la Iglesia (Ley 65, 1963, art. 10) por encima de los miembros de la alma mater. En este contexto surgió un movimiento estudiantil de grupos afiliados a la FUN o al Movimiento Obrero Estudiantil Campesino (MOEC) que no dudó en manifestar su inconformismo y en procurar la reivindicación de sus intereses gremiales y políticos. La respuesta del Gobierno consistió, como ocurrió en otros lugares del país, en acudir a la fuerza. Así, hacia 1971, en pleno estado de sitio, Mario Latorre no tuvo otra opción que renunciar a la rectoría, demostrando así el grado de inestabilidad en que había caído la institución 9 . De hecho, el Gobierno tuvo que otorgar a los rectores de las universidades oficiales, mediante el Decreto 1259 del 25 de junio de 1971, los poderes especiales que solo le eran atribuidos a los consejos superiores, lo cual en la práctica venía a suprimir el gobierno universitario al cerrar el órgano supremo de la autoridad universitaria, acto que quedó legalizado con la expedición del Decreto 2070 del 9 de octubre de 1971 (Secretaría General Universidad Nacional de Colombia, 1971a).

Mientras esto acontecía, la Asamblea General Estudiantil informaba sobre el paro nacional proyectado para el 21 y 22 de abril, en apoyo al Programa Mínimo. Con ello se pretendía presionar al Gobierno nacional para que aceptara los posibles acuerdos a que estudiantes y directivas llegaran en sus respectivas universidades (Administración Documental Universidad de Antioquia, 1971c). El Gobierno, sin embargo, reaccionó con el uso de la fuerza y ordenó el cierre indefinido de todas las

9 El 20 de enero fue nombrado como rector el profesor Diego López Arango tras aceptar la renuncia de Latorre; el 23 de abril renunció López y se encargó la dirigencia de la Universidad a Miguel Hernández (Secretaría General Universidad Nacional de Colombia, 1971b). 
El estudiantado colombiano y la apuesta por el cogobierno y la autonomía universitaria...

universidades del país mediante los Decretos $580^{10}$ y 581 . Las autoridades de las más importantes instituciones universitarias protestaron la medida, pues consideraban que a través de los diálogos la situación estaba llegando a buen término (Administración Documental Universidad de Antioquia, 1971d).

Ante la presión ejercida por los estudiantes y las autoridades universitarias, el gobierno del presidente Misael Pastrana no tuvo otra opción que formular las bases de una reforma a la universidad colombiana. Desde la óptica gubernamental, si bien se aceptaba que los elementos centrales de tal institución eran los profesores y los estudiantes, se consideraba que su participación en la dirección y orientación de la política universitaria no podía ser exclusiva, pues de ese modo la universidad quedaba desvinculada de la realidad social nacional (Pastrana presenta reforma universitaria, 1971). Luis Carlos Galán, en ese momento ministro de Educación, permitió la reapertura de las universidades, lo cual se dio una semana después, tras facultar a los rectores universitarios para que retomaran las actividades académicas cuando la situación de conflicto hubiese pasado (Autorizadas la reapertura de la U. de A. y de la UIS, 1971). Posteriormente, el mismo ministro tendría que revocar el Decreto 580, autorizando la reapertura de la Universidad Nacional (Catálogo General Universidad Nacional de Colombia, 1973).

La propuesta del Gobierno fue aprovechada por el movimiento estudiantil para ampliar sus demandas al plantear que, mientras la reforma gubernamental era elaborada, las universidades podían reemplazar los consejos superiores con un organismo provisional de mayoría profesoral y estudiantil. Pese a que varios rectores consideraron esta medida inadmisible, los diálogos en distintas universidades culminaron con la aprobación de una reforma sustancial de sus consejos superiores. En la Universidad Nacional de Colombia, la Universidad de Antioquia y la Universidad Industrial de Santander se acogió una fórmula de organización temporal en la

10 El Decreto 580 facultó al presidente y al ministro de Educación para suspender las tareas docentes y académicas de los centros de educación superior y de educación media a nivel nacional, departamental o municipal, cuando los estudiantes o profesores de dichos centros promovieran o realizaran, en el recinto de estos o en lugares públicos, actos que atentaran contra el orden público o dificultaran su restablecimiento, tales como paros temporales o indefinidos o asambleas que impidieran la vida académica normal. Igualmente, el Gobierno podía ordenar la cancelación de todos o algunos de los contratos de trabajo vigentes entre dichos centros y sus servidores, así como la de las demás relaciones de carácter laboral y estatutario. La participación en cualquiera de los dichos actos sería causal de cancelación de matrícula, terminación del contrato o destitución. 
que sus consejos estarían conformados por nueve miembros: tres representantes de los profesores, tres representantes de los estudiantes, el rector y dos representantes del Gobierno (Acevedo, 2016). Con esta fórmula se excluyeron los gremios y la Iglesia de los Consejos, aunque el rector continuaba teniendo un peso importante en el gobierno universitario ${ }^{11}$. Instalados los nuevos consejos, sus miembros levantaron las penas que las anteriores administraciones habían impuesto a los estudiantes y profesores movilizados en pro de la reforma. A su vez, se implementaron acciones administrativas en procura de atender las demandas académicas de los estudiantes y se realizó el nombramiento de funcionarios, con miras a restablecer la normalidad de las instituciones.

\section{Conclusiones}

El tema del gobierno en la universidad colombiana estuvo presente en el movimiento estudiantil desde los años veinte hasta principios de la década del setenta del siglo pasado. Aparejado a la defensa de la autonomía universitaria, la composición de los consejos superiores fue un asunto que interesó a estudiantes, profesores, rectores y sectores externos a la universidad. Dicho interés tuvo su raíz en los postulados liberales de la Reforma de Córdoba, momento en el que se pensó como necesaria la modernización de las casas de estudio para sacarlas del anquilosamiento en el que se encontraban. Aunque las luchas de los estudiantes colombianos se centraron inicialmente en cuestiones gremiales, la inquietud por conseguir mayor injerencia en las instancias de poder fue una de las formas como la politización permeó la movilización social por demandas académicas. La relación con los partidos políticos tradicionales tampoco fue obstáculo para que se reivindicara la autonomía universitaria y se esbozara un gobierno de los estudiantes y los profesores, actores de primer orden de la vida universitaria. La convicción que existía en el fondo de estas pretensiones consistía en asumirse como una especie de vanguardia intelectual que estaba llamada a organizar y determinar libremente los destinos de las instituciones universitarias.

11 La Universidad Industrial de Santander acogió la propuesta en el mes de abril de 1971 y la legalizó a través del Acuerdo 015; la Universidad de Antioquia, después de enredadas discusiones y acciones de presión, acogió la propuesta en enero de 1972 luego de la expedición por parte del Gobierno del Decreto 038 (Administración Documental Universidad de Antioquia, 1971e). 
Luego del giro hacia la izquierda, el estudiantado asumió la necesidad de incidir en el gobierno de las universidades e hizo de ello una causa importante en su proceder como actor social y político emergente. Ciertamente, por ser mayoría en los consejos superiores - junto con los profesores-, su lucha demandó esfuerzo y requirió organización, no solo para sostener la propuesta en escenarios propiamente estudiantiles sino para diferenciar su posición respecto a las iniciativas de reforma provenientes de las autoridades universitarias y del Ministerio de Educación. Existe un aspecto poco conocido del movimiento estudiantil de principios de los años setenta: el hecho de que el estudiantado coincidiera con las autoridades universitarias en la defensa de la autonomía y en la pertinencia de modificar las estructuras de poder en las universidades. Aunque se sabe que no hubo consenso entre los estudiantes por la causa del cogobierno debido al lugar que los diferentes grupos políticos le otorgaban a la universidad en el proceso de cambio social, lo relevante reside en constatar que su adopción en algunas universidades fue quizás el triunfo más importante de la movilización estudiantil en Colombia.

A pesar de ello, la victoria fue fugaz y poco trascendental para el destino de la universidad en el país. Efectivamente, la reforma orgánica no fue aprobada ni reconocida por el Estado, y todas las acciones emprendidas por los consejos transitorios fueron coyunturales, dado el estado de conmoción constante que experimentaban las universidades del país. Así pues, los estudiantes jamás pudieron ejercer de una manera clara el poder que tanto ansiaban. Ello no fue obstáculo para que esta causa articulara transitoriamente lo gremial y lo político en las filas estudiantiles, aunque la partida la ganó el radicalismo de izquierda que terminó por enviar a jóvenes a los grupos guerrilleros. Por su parte, las autoridades políticas y universitarias, en un contexto de anticomunismo y desconfianza de la movilización popular, cerraron las vías para acometer reformas a la estructura de poder de las universidades, imponiendo en los años siguientes a los llamados rectores policías. A diferencia de otros lugares en el continente, en Colombia sí hubo un cogobierno universitario. Sus limitados alcances - cuyas consecuencias se pueden observar hasta el día de hoy- permiten continuar hablando de estamentos universitarios, como si se viviera en el Antiguo Régimen. En el presente, además, las autoridades universitarias continúan siendo elegidas en procesos democráticos en los que todos los votos no tienen el mismo valor. 


\title{
Sobre los autores
}

\begin{abstract}
Álvaro Acevedo Tarazona. Historiador y magíster en Historia por la Universidad Industrial de Santander, Colombia; especialista en Filosofía por la Universidad de Antioquia, Colombia; doctor en Historia por la Universidad de Huelva, España. Tiene el Posdoctorado en Ciencias de la Educación por la Universidad Pedagógica y Tecnológica de Colombia, y actualmente es profesor titular de la Universidad Industrial de Santander. Áreas de interés: comunicación, educación y movimientos universitarios; proyectos educativos y construcción de memoria nacional; movimientos sociales, política y universidad: perspectivas comparadas e historia de la universidad en Colombia. Director del grupo de investigación Políticas, Sociabilidades y Representaciones Histórico-Educativas. Investigador Sénior (Minciencias). Ha publicado los libros La experiencia histórica del cogobierno en la Universidad Industrial de Santander: concepciones y divergencias en disputa por la autonomía universitaria, 1971-1976 (2016), Memorias de una época: el movimiento estudiantil en Colombia en los años sesenta y setenta del siglo xx (2016), 1968. Historia de un acontecimiento: utopía y revolución en la universidad colombiana (2017).
\end{abstract}

Andrés David Correa Lugos. Historiador y archivista por la Universidad Industrial de Santander, Colombia, y candidato a magíster en Historia por esta misma universidad. Miembro del grupo de investigación Políticas, Sociabilidades y Representaciones Histórico-Educativas. Áreas de interés: movimientos sociales contemporáneos; movimiento estudiantil; globalización como dinámica social del cambio; historia de los medios de comunicación; historia social de la ciencia. Investigador Junior (Minciencias). Ha publicado los capítulos de libros "El bucle de la indignación: un análisis a la identidad y la construcción del imaginario estudiantil colombiano” (2016), “Engranajes y gadgets: sobre cómo la tecnocracia y la globalización modifican la educación en América Latina” (2016), "Transiciones del movimiento estudiantil colombiano: de la utopía antisistémica al pragmatismo del procomún” (2018) y “Atajos y rendimiento en la universidad tardomoderna” (2018).

\section{Referencias}

\section{Fuentes primarias}

Administración Documental Universidad de Antioquia. (1971a, abril 6). Acta 1708 del Consejo Directivo. Archivo Universidad de Antioquia, Medellín.

Administración Documental Universidad de Antioquia. (1971b, abril 13). Acta 1709 del Consejo Directivo. Archivo Universidad de Antioquia, Medellín.

Administración Documental Universidad de Antioquia. (1971c, s. f.). Acta 1710 del Consejo Directivo. Archivo Universidad de Antioquia, Medellín.

Administración Documental Universidad de Antioquia. (1971d, abril 23). Acta 1712 del Consejo Directivo. Archivo Universidad de Antioquia, Medellín. 
El estudiantado colombiano y la apuesta por el cogobierno y la autonomía universitaria...

Administración Documental Universidad de Antioquia. (1971e, junio 22). Acta 1737 del Consejo Directivo. Archivo Universidad de Antioquia, Medellín.

Asociación Colombiana de Universidades-Consejo Nacional de Rectores. (1970a, abril 10). Carta dirigida a Fernando Hinestrosa Forero, ministro de Justicia y ministro de Educación encargado. (Rollo 002, f. 3). Archivo Universidad Industrial de Santander, Bucaramanga.

Asociación Colombiana de Universidades-Consejo Nacional de Rectores. (1970b, abril 10). Carta dirigida a Fernando Hinestrosa Forero, ministro de Justicia y ministro de Educación encargado. (Rollo 002, f. 5). Archivo Universidad Industrial de Santander, Bucaramanga.

Autorizadas la reapertura de la U. de A. y de la UIs. (1971, mayo 13). El Colombiano, p. 1.

Catálogo General Universidad Nacional de Colombia. (1973). Archivo Universidad Nacional de Colombia, Bogotá.

García Posada, J. (1971, abril 21). Cerrada la Universidad de Antioquia: también la Nacional de Medellín y la U. I. de Santander. El Colombiano, p. 1.

Ley 65 de 1963 (19 de diciembre de 1963), por la cual se establece el régimen orgánico de la Universidad Nacional de Colombia y se dictan otras disposiciones. Diario Oficial n.o 31.270. http://www.suin-juriscol.gov.co/viewDocument.asp?ruta=Leyes/1616770

Los “Hippies": sed, hambre y pantano en el Festival del Ancón. (1971, junio 20). El Colombiano.

Pastrana presenta reforma universitaria. (1971, mayo 5). El Colombiano, p. 24.

Secretaría General Universidad Nacional de Colombia. (1971a, octubre 9). Decreto 2070. Archivo Universidad Nacional de Colombia, Bogotá.

Secretaría General Universidad Nacional de Colombia. (1971b, s. f.). Decreto 66 de 1971 y Decreto 621 de 1971. Archivo Universidad Nacional de Colombia, Bogotá.

Uribe Ferrer, R. (1971, abril 11). La Iglesia no renunciará al Consejo Superior de la U. de A. El Colombiano, pp. 1 y 11.

\section{Fuentes secundarias}

Acevedo, Á. (2004). Modernización, conflicto y violencia en la universidad en Colombia: AUDESA, 1953-1984. Ediciones UIS.

Acevedo, Á. (2010). A cien años de la Reforma de Córdoba, 1918-2018: la época, los acontecimientos, el legado. Historia y Espacio, (36), 1-14.

Acevedo, Á. (2016). La experiencia histórica del cogobierno en la Universidad Industrial de Santander: concepciones y divergencias en disputa por la autonomía universitaria, 1971-1976. Ediciones UIS.

Acevedo, Á. (2017). 1968. Historia de un acontecimiento: utopía y revolución en la universidad colombiana. Ediciones UIS.

Acevedo, Á. y Correa,J. (2016). Tinta roja. Prensa, politica y educación en la República Liberal (19301946). El Diario de Pereira y Vanguardia Liberal de Bucaramanga. UIS-UTP-Colciencias. 
Acevedo, Á. y Samacá, G. (2013). Juventudes universitarias de izquierda en Colombia en 1971: un acercamiento a sus discursos ideológicos. Historia Caribe, 8(22), 195-229.

Acevedo, Á. y Villabona, J. (2015). El cogobierno en la Universidad de Antioquia, 1970-1973: una victoria del movimiento estudiantil y profesoral. Revista Historia y Espacio, (44), 145-167.

Archila, M. (1995). Protestas sociales en Colombia, 1946-1958. Historia Crítica, (11), 63-78.

Archila, M. (1999). Entre la academia y la política: el movimiento estudiantil y Colombia, 1920-1974. En R. Marsiske (Comp.), Movimientos estudiantiles en la historia de América Latina (Vol.1, pp. 158-174). Universidad Nacional Autónoma de México-Centro de Estudios sobre la Universidad-Plaza y Valdés.

Archila, M. y Cote, J. (2009). Auge, crisis y reconstrucción de las izquierdas colombianas (1958-2006). En M. Archila, J. Cote, Á. Delgado, M. C. García, P. Madariaga y Ó. H. Pedraza, Una historia inconclusa: izquierdas políticas y sociales en Colombia (pp. 55-75). Cinep-Colciencias.

Arias Trujillo, R. (2007). Los Leopardos: una historia intelectual de los años 1920. Ediciones Uniandes.

Barriga, J. (10 de diciembre de 2015). Festival de Ancón: sexo, drogas y rocanrol en el Woodstock criollo. Vice. https://www.vice.com/es_co/article/r7vjkz/festival-de-ancon-lostres-dias-en-los-que-colombia-fue-el-pais-mas-hippie-del-mundo

Borrero Cabal, A. (2008). La universidad: estudio sobre sus orígenes, dinámicas y tendencias. Editorial Pontificia Universidad Javeriana.

Buchbinder, P. (2005). Historia de las universidades argentinas. Sudamericana.

Cantón, D., Moreno, J. y Ciria, A. (2005). Argentina: la democracia constitucional y su crisis. Paidós.

Castro-Gómez, S., Hoyos G. y Millán C. (Eds.). (2007). Pensamiento colombiano del siglo xx (Vol. 2). Universidad Javeriana-Instituto Pensar.

Ciria, A. y Sanguinetti, H. (1987). La Reforma Universitaria (1918-1983). Centro Editor de América Latina.

Cote Rodríguez, J. (2009). El movimiento estudiantil de 1971: entre la homogeneidad y la heterogeneidad. En M. Archila, J. Cote, Á. Delgado, M. C. García, P. Madariaga y Ó. H. Pedraza, Una historia inconclusa: izquierdas políticas y sociales en Colombia (pp. 402-426). Cinep-Colciencias.

Finocchiaro, A. (2004). Estado Nacional: un estudio sobre autonomía universitaria. Prometeo.

Garzón, E. (2010). Rompiendo esquemas: discusiones, consignas y tropeles del estudiantado universitario en Colombia en 1971. Controversia, (194), 234-267.

Goethe, J. W. (1806). Fausto. Austral.

González, R. (2018). 1968: el nacimiento de un mundo nuevo. Debate. 
El estudiantado colombiano y la apuesta por el cogobierno y la autonomía universitaria...

Henderson, J. (2006). La modernización en Colombia: los años de Laureano Gómez, 1880-1965. Editorial Universidad de Antioquia.

Hernández, I. (2007). El programa mínimo de los estudiantes colombianos. Movimiento estudiantil universitario de 1971 por la universidad: todo un país. Revista Historia de la Educación Colombiana, (10), 29-57.

Herrera, V. y Trujillo, L. (1993). Movimiento estudiantil-Universidad del Valle-1971: como fuerza socialy su influencia histórica [Tesis de pregrado, Universidad del Valle, Cali, Colombia].

Jiménez, A. (2017). Una mirada al movimiento estudiantil colombiano. 1954-1978. Boletín Cultural y Bibliográfico, 51(93), 4-21. https://www.banrepcultural.org/boletin-cultural/ content/una-mirada-al-movimiento-estudiantil-colombiano-1954-1978

Latorre, M. y Murillo, G. (1984). Participación política, percepción política y liderazgo de la juventud colombiana: una percepción histórica. En M. Cárdenas y A. Díaz Uribe (Coords.), Juventudy política en Colombia (pp. 12-38). Fescol-sER.

Leal, F. (1984). La participación política de la juventud universitaria como expresión de clase. En M. Cárdenas y A. Díaz Uribe (Coords.), Juventud y política en Colombia (pp. 155-203). Fescol-SER.

Losada, R. y Vélez, E. (1984). La alienación política entre los jóvenes colombianos. En M. Cárdenas y A. Díaz Uribe (Coords.), Juventud y política en Colombia (pp. 12-38). Fescol-ser.

Moreno Durán, R. H. (1989). La memoria irreconciliable de los justos: la Universidad Nacional en la década de los 60. Análisis Político, (7), 77-87.

Moreno Martínez, O. (2009). El paro estudiantil de mayo de 1938. Anuario Colombiano de Historia Socialy de la Cultura, 36(2), 41-63.

Múnera, L. (2011). La Reforma de Córdoba y el gobierno de las universidades públicas en América Latina. Revista Ciencia Política, (12), 6-40.

Ocampo, J. (2008). Maestro Germán Arciniegas, el educador, ensayista, culturólogo e ideólogo de los movimientos estudiantiles en Colombia. Revista Historia de la Educación Latinoamericana, (11), 13-58.

Ordóñez, L. A. (2007). Universidad del Valle 60 años 1945-2005: atando cabos en clave de memoria. Editorial Universidad del Valle.

Palacios, M. (2012). Violencia pública en Colombia, 1958-2010. Fondo de Cultura Económica.

Pastrana, E. (2008). La reforma universitaria, el movimiento de Córdoba y sus repercusiones en Colombia. Educere, (41), 313-318.

Programa Mínimo del Movimiento Nacional Estudiantil. (1971). En (s. d.), Crisis universitaria colombiana 1971: itinerario y documentos. Ediciones El Tigre de Papel.

Pulido, D. A. (2010). "Vanguardia juvenil o elitismo estudiantil: el lugar de la intelectualidad universitaria colombiana en América Latina durante los años veinte. Apuntes para una aproximación comparada" [En: Memorias del IV Seminario Taller Internacional 
Vendimia 2010: “Construcción de Nación: la Universidad del Futuro en Iberoamérica”, Villa de Leyva, Colombia].

Quiroz, C. (2002). La Universidad Nacional de Colombia en sus pasillos. Editorial un.

Radio Macondo (25 de diciembre de 2018). Rock and roll en Colombia: el impacto de una generación en la transformación cultural del país en el siglo xx. Radio Macondo. https://www. radiomacondo.fm/arte-y-cultura/rock-and-roll-en-colombia-el-impacto-de-una-generacion-en-la-transformacion-cultural-del-pais-en-el-siglo-xx/

Rébora, L. (1989). La reforma universitaria 1918-1988. Legasa.

Ruiz, M. (2002). Sueños y realidades, procesos de organización estudiantil, 1954-1966. Editorial UN.

Sader, E., Gentili, P. y Aboites, H. (2008). La Reforma Universitaria. Desafíos y perspectivas noventa años después. Clacso.

Salgado, S. A. (2015). Aportes para una historia de los movimientos estudiantiles en Colombia a través de sus publicaciones periódicas (1917-1929). En Á. Acevedo Tarazona, S. Sánchez Parra y G. Samacá Alonso (Comps.), ¡A estudiar, a luchar!: movimientos estudiantiles en Colombia y México, siglos xх y XxI (pp. 17-40). Editorial Universidad Autónoma de Sinaloa.

Samacá, G. y Calderón, S. (2015). Posibilidades interpretativas y nuevas fuentes para pensar la historia de la acción colectiva estudiantil en Colombia en los años setenta. En Á. Acevedo Tarazona, S. Sánchez Parra y G. Samacá Alonso. (Comps.), iA estudiar, a luchar!: movimientos estudiantiles en Colombia y México, siglos XX y XXI (pp. 83-114). Editorial Universidad Autónoma de Sinaloa.

Santamaría, R., Vélez, E. y Silva, G. (1984). La juventud universitaria y el sistema político: ¿caminos divergentes? En M. Cárdenas y A. Díaz Uribe (Coords.), Juventud y política en Colombia (pp. 102-126). Fescol-SER.

Scott, M. B. y Lyman, S. M. (1974). La rebelión de los estudiantes. Paidós.

Tirado, Á. (2014). Los años sesenta en Colombia: una revolución en la cultura. Debate.

Vaccarezza, L. (2006). Autonomía universitaria, reformas y transformación social. Clacso. 